\title{
Targeting intrusive imagery using a competing task technique: a case study
}

\author{
Lalitha Iyadurai $^{1, \star(D)}$, Susie A. Hales ${ }^{2}$, Simon E. Blackwell ${ }^{3}$, Kerry Young ${ }^{4}$ and Emily A. Holmes ${ }^{5,6}$ \\ ${ }^{1}$ Department of Psychiatry, University of Oxford, Oxford, UK, ${ }^{2}$ Oxford Institute of Clinical Psychology Training, University of \\ Oxford, Oxford, UK, ${ }^{3}$ Mental Health Research and Treatment Center, Ruhr-Universität Bochum, Bochum, Germany, \\ ${ }^{4}$ Woodfield Trauma Service, Central and North West London NHS Foundation Trust, London, UK, ${ }^{5}$ Department of \\ Psychology, Uppsala University, Uppsala, Sweden and ${ }^{6}$ Department of Clinical Neuroscience, Karolinska Institutet, \\ Stockholm, Sweden \\ *Corresponding author. Email: lalitha.iyadurai@psych.ox.ac.uk
}

(Received 7 May 2019; revised 3 March 2020; accepted 27 March 2020; first published online 29 June 2020)

\begin{abstract}
Background: Even in cases with complexity, simple techniques can be useful to target a specific symptom. Intrusive mental images are highly disruptive, drive emotion, and contribute to maintaining psychopathology. Cognitive science suggests that we might target intrusive images using competing tasks. Aims: We describe an imagery competing task technique within cognitive behavioural therapy (CBT) with a patient with bipolar disorder and post-traumatic stress disorder (PTSD) symptoms. The intervention including Tetris computer game-play - was used (1) to target a specific image within one therapy session, and (2) to manage multiple images in daily life.

Method: A single case (AB) design was used. (1) To target a specific image, the patient brought the image to mind and, after mental rotation instructions and game-play practice, played Tetris for 10 minutes. Outcomes, pre- and post-technique, were: vividness/distress ratings when the image was brought to mind; reported intrusion frequency over a week. (2) To manage multiple images, the patient used the intervention after an intrusive image occurred. Outcomes were weekly measures of: (a) imagery characteristics; (b) symptoms of PTSD, anxiety, depression and mania.

Results: (1) For the target image, there were reductions in vividness ( $80 \%$ to $40 \%)$, distress $(70 \%$ to $0 \%$ ), and intrusion frequency (daily to twice/week). (2) For multiple images, there were reductions from baseline to follow-up in (a) imagery vividness (38\%), realness (66\%) and compellingness (23\%), and (b) PTSD symptoms (Impact of Events Scale-Revised score 26.33 to 4.83 ).

Conclusion: This low-intensity intervention aiming to directly target intrusive mental imagery may offer an additional, complementary tool in CBT.
\end{abstract}

Keywords: anxiety; bipolar disorder; cognitive science; imagery-focused cognitive therapy; intrusions; mental imagery; PTSD; single-case experimental design

\section{Introduction}

Vivid, emotional, recurrent, intrusive mental images feature in a wide range of psychological disorders. These images can be highly distressing and disrupt patients' daily lives, presenting a treatment target in their own right (Iyadurai et al., 2019). Furthermore, intrusive images may be a driving symptom in the maintenance of some disorders, such as post-traumatic stress disorder (PTSD) and bipolar disorder (e.g. Hales et al., 2018). Techniques to target intrusive images may alleviate distress, improve functioning and have downstream benefits for treating psychological disorders.

\footnotetext{
(c) British Association for Behavioural and Cognitive Psychotherapies 2020. This is an Open Access article, distributed under the terms of the Creative Commons Attribution licence (http://creativecommons.org/licenses/by/4.0/), which permits unrestricted re-use, distribution, and reproduction in any medium, provided the original work is properly cited.
} 
Established techniques to target intrusive imagery in cognitive behavioural therapy (CBT), such as imaginal reliving and imagery rescripting, involve detailed discussion of imagery content and meaning. This prospect can feel frightening for some patients. Furthermore, these techniques address single images, and may not be optimal when patients experience multiple intrusive images or images with frequently changing content. Metacognitive techniques can help patients disengage from images, but can be challenging to use when images are very powerful or compelling. In these circumstances, simple, low-intensity techniques to disrupt intrusive images would offer a useful additional tool in CBT.

A potential novel approach developed from basic cognitive science research involves using cognitive tasks that tax visuospatial working memory to directly target imagery characteristics rather than content or meaning. Tasks such as spatial pattern tapping and bilateral eye movements have been found to reduce the vividness and emotionality of distressing mental images (Van den Hout and Engelhard, 2012) as well as the frequency with which they intrude in daily life (Kessler et al., 2020). A procedure with greater potential ecological validity - a brief behavioural intervention including playing the popular visuospatial computer game 'Tetris' - has been shown to reduce the frequency of intrusive images over the subsequent week relative to a control procedure or no task, in both laboratory studies (e.g. Kessler et al., 2020) and after real-life trauma (e.g. Iyadurai et al., 2018). This approach could offer a simple, low-intensity and non-threatening technique in CBT to directly target the properties of intrusive images, irrespective of image content.

Here we describe the use of this novel technique in CBT - a simple intervention procedure including playing the visuospatial computer game Tetris - to target intrusive imagery as part of a formulation-driven imagery-focused treatment for mood instability (Holmes et al., 2019) with a patient with bipolar disorder and co-morbid PTSD symptoms. The technique was used with two different applications: (1) to target the vividness, distress and frequency of a specific problematic image within a single therapy session, and (2) to disrupt various problematic images as an adaptive strategy in day-to-day life over the treatment period.

\section{Method}

\section{Case presentation}

Ruth (pseudonym) was referred for outpatient psychological treatment by her psychiatrist, following a diagnosis of bipolar II disorder. At assessment, using the Structured Clinical Interview for DSM-IV-TR (SCID), she was currently euthymic but reported ongoing fluctuations in mood and energy levels, which affected her ability to work and engage in daily routines consistently. She additionally had PTSD symptoms and generalised anxiety disorder. She was currently taking three types of medication for mood management.

Ruth reported that her main problem was recurrent intrusive images with varying content, including 'flashback' images of traumatic events in her childhood, 'flashforward' images of anxiety-provoking social situations and 'obsessive' images related to security at home. She experienced the images as highly vivid and detailed, hence very real and compelling. Consequently, she felt distressed, anxious and often guilty, and found the images affected her sleep, concentration and work. She avoided certain social situations and often spent hours checking her home. Following an imagery micro-formulation that linked these factors, we agreed to target her recurrent images in treatment, with the aim of reducing anxiety and stabilising mood. A visuospatial task intervention was selected as one suitable treatment technique, (1) to specifically target image vividness/realness, hypothesised to drive the impact on anxiety and mood, and (2) as it could be used to target multiple images with different content. 


\section{Measures}

(1) Targeting a specific image within session (pre- and post-technique outcomes)

Vividness and distress ratings of the image when brought to mind (0-100\%); reported intrusion frequency of the image over the last week (number of days on which the image intruded).

(2) Managing multiple images in daily life (weekly measures)

(a) Imagery characteristics during the past week. Visual analogue scale (VAS) ratings of imagery realness, vividness, and compellingness $(0=$ not at all to $10=$ extremely); subjective distress of intrusions assessed using the Impact of Events Scale-Revised (IES-R) intrusion subscale score.

(b) Symptoms of PTSD, anxiety, depression and mania during the past week. Impact of Events Scale-Revised (IES-R); Beck Anxiety Inventory (BAI); Quick Inventory of Depression Symptoms (QIDS); Altman Self-Rating Mania Scale (ASRM).

\section{Treatment}

\section{Overview}

Seven 1-hour sessions of imagery-focused cognitive therapy (Holmes et al., 2019) took place over an 11-week treatment period. There was a 3-week baseline period and 6-week follow-up period. The imagery-competing intervention was delivered in treatment session 2 .

(1) Targeting a specific image within session

Ruth brought to mind an image that had been particularly frequent and distressing in the last week, and rated it for vividness and distress. After a few minutes - during which the computer game Tetris (Marathon version) was loaded on a Nintendo DS (a small hand-held gaming device), the clinician (L.I.) explained the instructions including mental rotation, and Ruth had game-play practice - she played Tetris for 10 minutes (for further details, see Holmes et al., 2019, chapter 11). She then rated image vividness and distress again, and monitored its frequency over the subsequent week.

(2) Managing multiple images in daily life

Ruth downloaded Tetris on her smartphone, and was encouraged to use the intervention when feasible (including playing Tetris for at least 10 minutes) after an intrusive image occurred in her day-to-day life.

\section{Results}

(1) Targeting a specific image within session

Ruth brought to mind an image of 'everyone looking at me, waiting for me to say something, and I can't get the words out'. She reported that the image had intruded daily over the last week, and rated it to be $80 \%$ vivid and $70 \%$ distressing when she brought it to mind. After the brief behavioural intervention including playing Tetris, she brought the image to mind again within the same session, and reported it was 'vague and dim' (40\% vivid: $50 \%$ reduction), was no longer distressing ( $0 \%$ distressing: $100 \%$ reduction), and that it 'doesn't feel real, like a painting'. On returning the next session, she reported that the image had only intruded on 2 days over the last week, and rated it to be $50 \%$ vivid and $30 \%$ distressing when she brought it to mind in the session.

(2) Managing multiple images in daily life

Ruth reported particularly compelling intrusive images in the evenings, which prevented her falling asleep. This left her tired during the day, and affected her work. She found that using the intervention at bedtime helped to disrupt the images and allowed her to get to sleep. Ruth used this technique as needed throughout treatment and follow-up. 

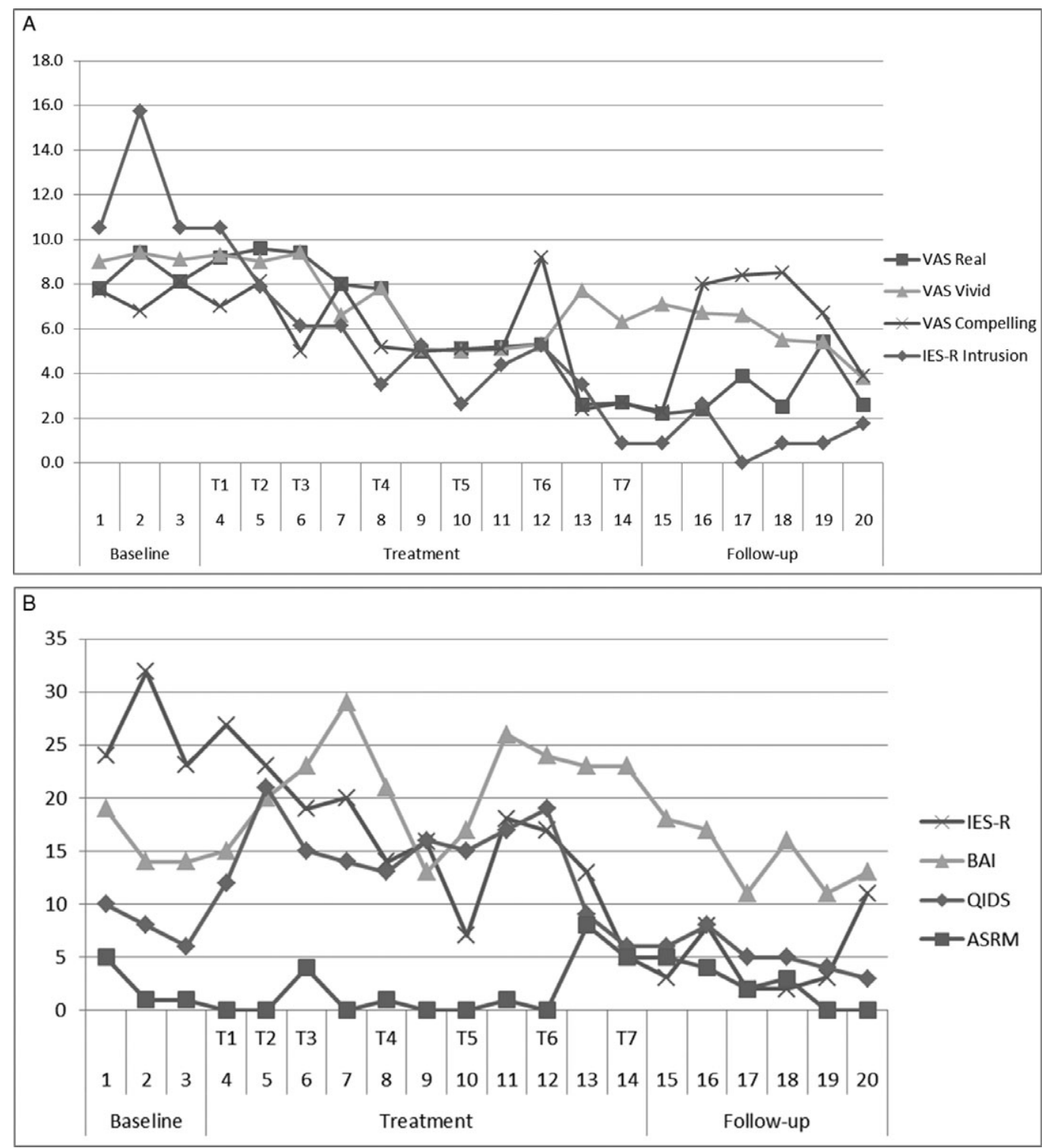

Figure 1. Weekly measures of (A) imagery characteristics and (B) symptoms of PTSD, anxiety, depression and mania over baseline, treatment and follow-up periods. T1 to T7 indicate the timing of the seven treatment sessions, with in-session use of the imagery-disrupting task taking place in treatment session 2. VAS, visual analogue scale (rated 0 to 10 ); IES-R Intrusion, Impact of Event Scale-Revised intrusion subscale (indicating intrusion distress); IES-R, Impact of Event Scale-Revised total score (including intrusion subscale); BAI, Beck Anxiety Inventory; QIDS, Quick Inventory of Depression Symptoms; ASRM, Altman Self-Rating Mania Scale.

\section{(a) Imagery characteristics}

Figure 1A shows a reduction from baseline to follow-up periods in mean weekly VAS ratings of image realness (mean 8.43 to 2.87: 66\% reduction), vividness (mean 9.17 to $5.73: 38 \%$ reduction), and compellingness (mean 7.53 to $5.77: 23 \%$ reduction). There was a reduction in mean IES-R intrusion subscale score, indicating distress from intrusions, from baseline (12.25) through treatment (5.09) to follow-up (1.12), with a statistically reliable change from baseline to follow-up (reliable change index $=-29.48$ ). 
(b) Symptoms of PTSD, anxiety, depression and mania

Mean total IES-R score reduced from baseline (26.33) through treatment (16.27) to follow-up (4.83), demonstrating a statistically reliable change from baseline to follow-up (reliable change index $=-76.01)$. At follow-up, Ruth no longer exhibited any PTSD symptoms during the SCID interview. Anxiety (BAI score) was 'minimal' at baseline (15.67) and follow-up (14.33), with a slight increase to 'moderate' during treatment (21.27). QIDS scores indicated 'mild' depression at baseline (8.00), 'moderate' depression during treatment (14.27), and a drop to 'normal' at follow-up (5.17). Mania scores (ASRM) remained subclinical throughout baseline (2.33), treatment (1.73) and follow-up (2.33). For measures other than the IES-R there was no reliable change from baseline to follow-up. Figure 1B shows weekly scores on these outcome measures across the baseline, treatment and follow-up periods.

\section{Ruth's experience}

Ruth reported that her use of the imagery-competing technique became more automatic throughout treatment. At the end of treatment she felt more in control of her images and her mood, and realised that 'having an image did not mean it was real'. She reported increased self-confidence in work and social events and was less avoidant of some social situations.

\section{Discussion}

This case study demonstrates the use of a simple, novel intervention technique within CBT, distilled from basic cognitive science, with a patient with bipolar disorder and comorbid PTSD symptoms. The technique is a brief intervention including playing the visuospatial computer game Tetris to target a highly disruptive symptom - intrusive mental imagery. Results provide a preliminary indication that the technique can be used within session to reduce the vividness and emotionality of a selected image, as well as the frequency with which it intrudes over the subsequent week, consistent with preceding cognitive science studies (e.g. Kessler et al., 2020). Furthermore, use of the technique as an adaptive strategy to disrupt multiple images in daily life was associated with reductions in ratings of imagery realness, vividness and compellingness, and distress from intrusions. The patient's subjective account indicated that using the technique led to her imagery feeling 'vague and dim' and 'like a painting', and to the understanding that 'having an image did not mean it was real'. These changes in image quality and appraisal appeared to be linked with the reduction in her distress.

A corresponding reduction in IES-R scores from baseline to follow-up suggested that targeting imagery may have had downstream beneficial effects on psychological symptoms, consistent with theoretical models of imagery in psychological disorders and recent case series (e.g. Hales et al., 2018; Holmes et al., 2016). Anxiety, depression and mania symptom scores were low at baseline. Slight increases in anxiety and depression symptoms during treatment coincided with a family fall-out and increased work pressure.

The intervention technique including computer game-play may offer a simple, low-stigma and engaging addition to traditional cognitive techniques (e.g. imagery rescripting, imaginal reliving, metacognitive approaches) by directly targeting the properties of intrusive images instead of addressing their content and meaning. This may be particularly useful when formulation indicates that other approaches are not suitable as a first treatment option, or when the patient experiences multiple, highly compelling images that are difficult to disengage from, as in this case. Whilst this patient used the visuospatial computer game Tetris as part of the intervention, other visuospatial tasks may be effective, including activities the person already finds engaging, e.g. doing art or jigsaw puzzles. However, these other tasks have not yet been tested in experimental studies. 
In this study, we did not seek to evaluate the specific impact of the imagery-targeting technique on overall outcome, as it was used as part of a broader imagery-focused CBT treatment. Other imagery-focused techniques within the therapy, as well as other unknown confounders, may have impacted the outcome. Further research to evaluate the technique might involve a multiplebaseline design using different target images, and diary-based assessment of daily intrusion frequency, vividness and distress.

Supplementary material. To view the extended report for this article, please visit https://doi.org/10.1017/S135246 5820000296

Acknowledgements. We would like to thank 'Ruth' for giving us permission to publish an account of her work with us, and the OxMAPP clinical team for peer supervision during this work.

Financial support. This work was supported by the National Institute of Health Research (to L.I., grant number NIHR-DRF2011-04-076) and the Wellcome Trust (to E.A.H., grant number WT088217; also supporting S.A.H. and K.Y.).

Conflicts of interest. S.A.H., K.Y. and E.A.H. have published a book entitled Imagery-Based Cognitive Therapy for Bipolar Disorder and Mood Instability (Holmes et al., 2019). L.I. and S.E.B. have no conflicts of interest with respect to this publication.

Ethics statements. This work was conducted in line with the Ethical Principles of Psychologists and Code of Conduct as set out by the American Psychological Association (2017). The work described in this case study was part of the patient's routine clinical care. The patient provided written, informed consent for the authors to submit an anonymised account of our work with them for publication as a case study in a scientific journal. Any information which could potentially identify the patient has been removed or disguised.

\section{References}

Hales, S. A., Di Simplicio, M., Iyadurai, L., Blackwell, S. E., Young, K., Fairburn, C. G., . . \& Holmes, E. A. (2018). Imagery-focused cognitive therapy (ImCT) for mood instability and anxiety in a small sample of patients with bipolar disorder: a pilot clinical audit. Behavioural and Cognitive Psychotherapy, 46, 706-725. doi: 10.1017/s1352465818000334

Holmes, E. A., Bonsall, M. B., Hales, S. A., Mitchell, H., Renner, F., Blackwell, S. E., ... \& Di Simplicio, M. (2016). Applications of time-series analysis to mood fluctuations in bipolar disorder to promote treatment innovation: a case series. Translational Psychiatry, 6, e720. doi: 10.1038/tp.2015.207

Holmes, E. A., Hales, S. A., Young, K., \& Di Simplicio, M. (2019). Imagery-Based Cognitive Therapy for Bipolar Disorder and Mood Instability. New York, NY, USA: Guilford Press.

Iyadurai, L., Blackwell, S. E., Meiser-Stedman, R., Watson, P. C., Bonsall, M. B., Geddes, J. R., . . \& \& Holmes, E. A. (2018). Preventing intrusive memories after trauma via a brief intervention involving Tetris computer game play in the emergency department: a proof-of-concept randomized controlled trial. Molecular Psychiatry, 23, 674-682. doi: 10.1038/mp.2017.23

Iyadurai, L., Visser, R. M., Lau-Zhu, A., Porcheret, K., Horsch, A., Holmes, E. A., \& James, E. L. (2019). Intrusive memories of trauma: a target for research bridging cognitive science and its clinical application. Clinical Psychology Review, 69, 67-82. doi: 10.1016/j.cpr.2018.08.005

Kessler, H., Schmidt, A.-C., James, E. L., Blackwell, S. E., von Rauchhaupt, M., Harren, K., ... Holmes, E. A. (2020). Visuospatial computer game play after memory reactivation delivered three days after a traumatic film reduces the number of intrusive memories of the experimental trauma. Journal of Behavior Therapy and Experimental Psychiatry, 67, 101454. doi: 10.1016/j.jbtep.2019.01.006

Van den Hout, M. A., \& Engelhard, I. M. (2012). How does EMDR work? Journal of Experimental Psychopathology, 3, 724-738. doi: $10.5127 /$ jep.028212

Cite this article: Iyadurai L, Hales SA, Blackwell SE, Young K, and Holmes EA (2020). Targeting intrusive imagery using a competing task technique: a case study. Behavioural and Cognitive Psychotherapy 48, 739-744. https://doi.org/10.1017/ S1352465820000296 